\title{
Apparent Diffusion Coefficient Value Changes of Normal Uterine Cervix with Aging
}

\author{
Funda Dinç Elibol (iD ${ }^{1,}{ }^{*}$ and Sezen Bozkurt Köseoğlu (iD ${ }^{2}$ \\ ${ }^{1}$ Department of Radiology, Mugla Sitki Kocman University Hospital, Mugla, Turkey \\ ${ }^{2}$ Department of Obstetrics and Gynecology, Mugla Sitki Kocman University Hospital, Mugla, Turkey \\ "Corresponding author: Department of Radiology, Mugla Sitki Kocman University Hospital, Mugla, Turkey. Email: fundadi@yahoo.com
}

Received 2018 November 17; Revised 2019 August 21; Accepted 2019 August 25.

\begin{abstract}
Background: In the magnetic resonance imaging (MRI) of many tissues, apparent diffusion coefficient (ADC) values vary with age. In the literature, there are no studies in which normal cervix ADC values and their relationship with age is analyzed.

Objectives: To evaluate whether changes occur in ADC values with aging in the uterine cervix with 3-Tesla MRI.

Patients and Methods: Female patients aged between 18 and 70 years who were found to have lower abdominal diffusion-weighted magnetic resonance imaging(DWI) in the radiology database from December 2017 to May 2018 were evaluated. All DWI examinations were performed using a 3-T MR with $2 \mathrm{~b}$ values $\left(\mathrm{b}=50,800 \mathrm{~s} / \mathrm{mm}^{2}\right)$. A total of 161 patients were divided into two groups according to their menopausal status (premenopausal $n=106$, postmenopausal $n=55$ ). To evaluate the correlation between the age of the patient and the ADC values, Pearson correlation analysis was performed.

Results: The mean age of the patients was $41.4 \pm 11.6$ years. The mean ADC value was $1.276 \pm 0.25 \mathrm{~mm}^{2} / \mathrm{sec} \times 10^{-3}$ in the whole population. There was a negative correlation between age and ADC values. The mean cervix ADC value of premenopausal and postmenopausal patients was $1.333 \pm 0.23 \mathrm{~mm}^{2} / \mathrm{sec} \times 10^{-3}$ and $1.165 \pm 0.25 \mathrm{~mm}^{2} / \mathrm{sec} \times 10^{-3}$, respectively. There was a statistically significant difference between the groups in term of cervix ADC value $(\mathrm{P}<0.05)$.

Conclusion: A negative correlation between aging and ADC values was found. Menopausal status has an effect on the ADC values of the cervix. These findings may indicate cellular and extracellular matrix changes in the cervix tissue with aging and menopausal status.
\end{abstract}

Keywords: Aging, Uterine Cervix, Apparent Diffusion Coefficient

\section{Background}

Diffusion-weighted magnetic resonance imaging (DWI) is a noninvasive imaging technique that provides information on water proton mobility. This can make subtle microscopic abnormalities in tissue more obvious, thereby providing good tissue characterization. It is sensitive to the diffusion of water molecules in tissues, resulting in information on the extracellular space, tissue cellularity, and cellular membrane integrity (1).

The main initial clinical application of DWI was to detect acute cerebral ischemia in the brain and afterward expanded to detect several brain disorders (2). DWI was used to reveal microscopic details of tumor-like cellular density and proliferative activity (3-5). DWI can be used as a tool for characterizing microstructure of the tissue and some studies proposed that DWI might help to differentiate malignant and benign lesions in such tissues such as breast or thyroid $(6,7)$. It has also been shown that DWI with ad- vanced sequence designs has the potential to distinguish between differentiated and undifferentiated subtypes of thyroid carcinoma (8).

Today, DWI is used in the detection and characterization of malignancies, as well as in the evaluation of treatment success and detection of recurrence in abdominal malignancies (9). DWI has become a routine sequence in female pelvis imaging and is also used widely in uterine cervical cancer. The main uses of DWI in the uterine cervix are tumor detection, staging, lymph node differentiation, and treatment evaluation (9-16). Apparent diffusion coefficient (ADC) values enable the differentiation of cancer tissue from normal cervical tissue with high diagnostic accuracy (11). In addition, the potential ability of DWI to indicate histologic subtypes of cervix cancer has been shown $(10,11$, 14). ADC values of cervical tumors can represent tumor cellularity, which provides for the evaluation of histologic tumor grade through radiologic imaging (14-17). Lower ADC 
values may be related to poor prognosis (11). Pre-treatment ADC values were found lower in patients with complete response after treatment when compared with partial response (12). When evaluating the strength of the magnetic field, it has also been shown that 3 Tesla (3T) MRI contributes a significant benefit in detecting local and distant metastasis of cervical carcinoma then 1.5 T MRI $(17,18)$.

There are several studies of age-related ADC value changes in the normal appearance of several normalappearing tissues such as the prostate, vertebra, and muscles (19-21). In children, age and vertebral body leveldependent differences in ADC values occur due to varying composition and cellularity (20). ADC values of both the peripheral zone and central gland of the prostate gland increase with age (21). As our knowledge in literature, there is no study about age and normal cervix ADC values.

\section{Objectives}

The aim of this study was to evaluate if there was a correlation between ADC values and age for the uterine cervix with 3T MRI.

\section{Patients and Methods}

This study was conducted in the Department of Radiology of a tertiary hospital. Electronic records from December 2017 to May 2018 were analyzed retrospectively. Patients with lower abdomen MRI and DWI for various reasons were screened and compiled from the radiology archive. The cervicovaginal smear results of the patients were evaluated from the archive. Patients with both upper and lower abdominal MRI and only lower abdominal MRI were evaluated. Among 655 women who underwent lower abdominal MRI and DWI, 161 women met the inclusion criteria: aged between 18 and 71 years, and had a normal cervicovaginal smear. Patients with gynecologic malignancy, extra-gynecologic abdominal malignancy, and cervical mass, abnormal cervical cytology $(n=4)$, numerous nabothian cysts $(n=3)$ and those who had undergone hysterectomy ( $n=98)$ were excluded from the study. A total of 161 patients were divided into two groups according to their menopausal status. Menopause was accepted as 1 year without menstrual bleeding; all of the patients had experienced menopause naturally. A total of 106 patients were in the premenopausal period, and 55 patients were in the postmenopausal period.

\subsection{Radiologic Evaluation}

All DWI examinations were performed using a 3T MR (Siemens Magnetom Skyra, Erlangen, Germany) using transverse diffusion-weighted echo-planar imaging sequence. The ADC values were calculated from two different b values (b50 and b800 seconds $/ \mathrm{mm}^{2}$, repetition time [TR] / echo time [TE]: 6800/46, slice thickness/gap: $4 \mathrm{~mm} / 1 \mathrm{~mm}$, echo time: $31 \mathrm{~ms}$, field of view: $280 \mathrm{~mm} \times 270 \mathrm{~mm}$ ) and ADC maps were reconstructed with these values. The mean ADC values were calculated by manual placement of circular regions of interest (ROIs). The ROI diameter was the largest (with minimum $4 \mathrm{~mm}$ diameter of ROI), thus it was possible to exclude the cervical stroma and mucosa (Figure 1). To increase measurement accuracy on ADC maps, ADC maps were matched with the T1-weighted sequences. The ADC values of the cervix were measured three times on the axial ADC maps in the slice of where the cervix was seen most voluminous by the same researcher (10 years of experience). All measured ADC values were recorded as a mean \pm standard deviation for each measurement. Then, the mean value of the measurements was calculated for each patient by dividing the sum of ADC values by three. $\mathrm{ADC}$ values of the cervix were measured 3 weeks later by the same researcher another three times and the mean value of the ADCs was calculated to evaluate intraobserver variability.

\subsection{Statistical Analysis}

The SPSS statistics for Windows version 17.0 was used (SPSS Inc., Chicago, Ill, USA). The mean and standard deviation (SD) values of parameters were used to describe scale variables. Before analyzing the differences, the Kolmogorov-Smirnov test was used to define the normality of the parameters. Since the variables were found to be normally distributed due to Kolmogorov-Smirnov test, the independent samples $t$-test was used. To evaluate the relationship between variables because the variables were normally distributed, Pearson's correlation test was used. The receiver operator characteristics (ROC) curve analysis was used to establish the cut-off point for the ADC value. To determine the locally appropriate cut-off value for the highest Youden index (sensitivity + specificity - 1) was considered as the optimal cut-off value. Intraobserver variability for cervix ADC measurements were analyzed by calculating the interclass correlation coefficient (ICC: $0.00-0.20=$ poor correlation, $0.21-0.40=$ fair, $0.42-0.60=$ moderate, $0.61-0.80=$ good, and $0.81-1.00=$ excellent $)$. P values $<$ 0.05 were considered statistically significant. 

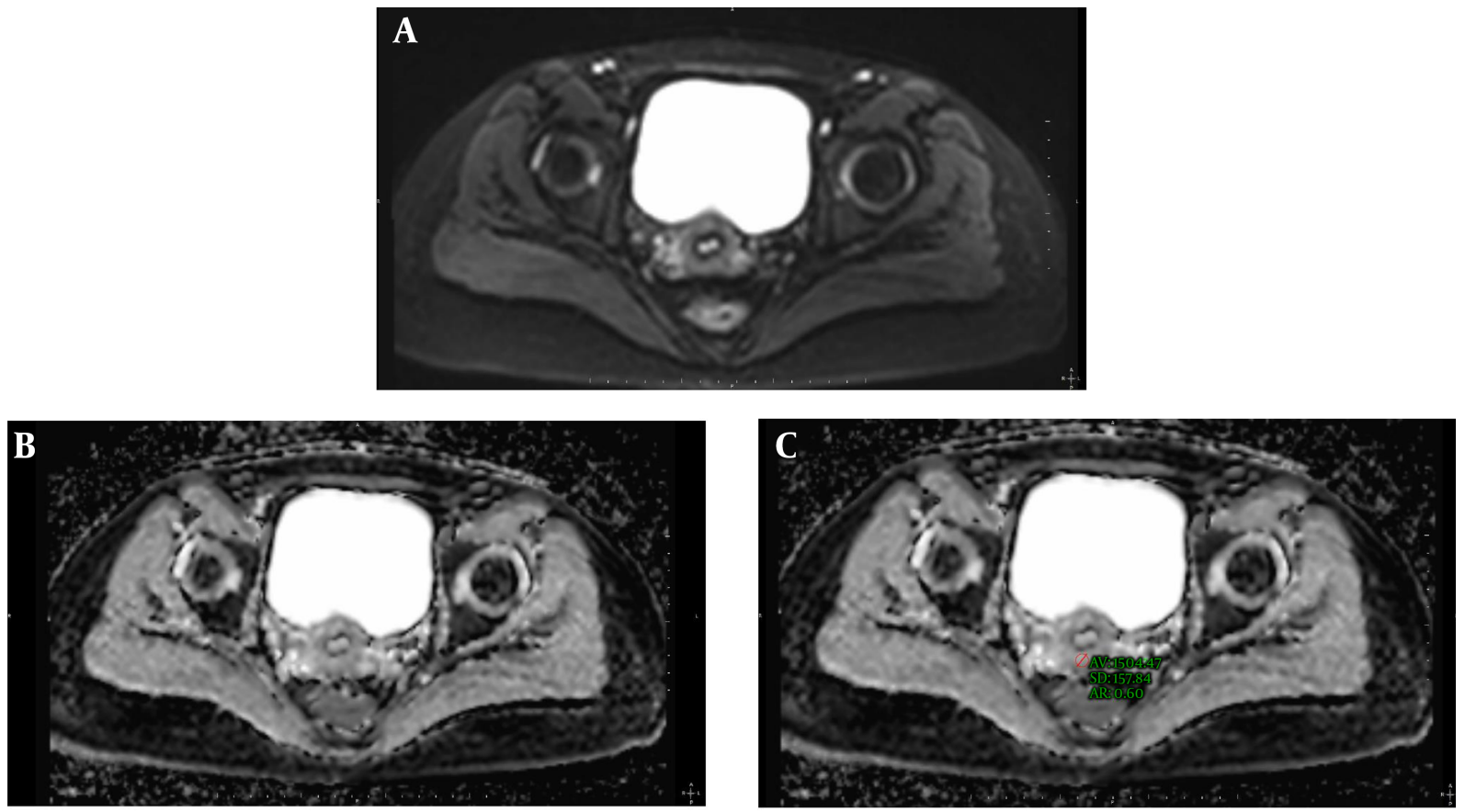

Figure 1. A, Patients b50 image at the level of the cervix. B, Apparent diffusion coefficient (ADC) map of the same slice. C, Measurement with regions of interest (ROI) of ADC value of cervix is seen.

\section{Results}

A total of 161 patients were included in the study. The mean age was $41.4 \pm 11.6$ years. The mean ADC value was $1.276 \pm 0.25 \mathrm{~mm}^{2} / \mathrm{sec} \times 10^{-3}$. The age and cervix ADC value were inversely correlated $(\mathrm{r}=-0.382, \mathrm{P}<0.001)$ (Figure 2$)$.

Patients were divided into two groups according to

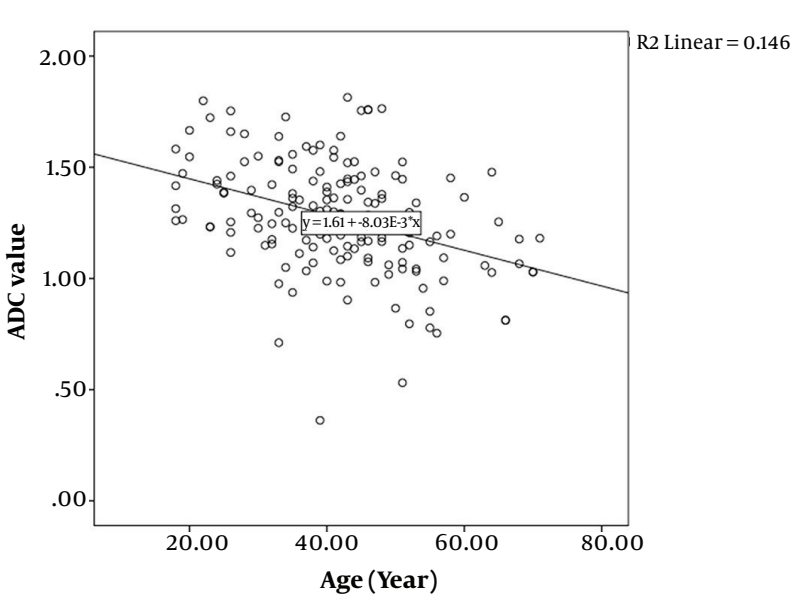

$\overline{\text { Figure 2. Scatter plot showing correlation between apparent diffusion coefficient }}$ (ADC) values and age. their menopausal status. The first group consisted of 106 premenopausal patients (mean age, $34.82 \pm 7.7$ ) and the second group consisted of 55 postmenopausal patients (mean age, $54.10 \pm 7.4$ ). The mean cervix ADC value was higher for premenopausal patients than for postmenopausal patients. There was a statistically significant correlation between the two groups in terms of cervix ADC value $(\mathrm{P}<0.001)$ (Table 1$)$. The area under the curves (AUC) for ADC was 0.710 (Figure 3). The cut-off level for cervix ADC was $1.234 \mathrm{~mm}^{2} / \mathrm{sec} \times 10^{-3}$, with a sensitivity and specificity of $67.3 \%$ and $67.9 \%$, respectively.

The ICC was calculated as $\mathrm{r}=0.78$ (between $0.469-0.917$ with $95 \%$ confidence intervals). The correlation was found as good for cervix ADC measurements $(\mathrm{P}<0.001)$ in our study.

Table 1. Comparison of Age and ADC Values Between Premenopausal and Postmenopausal Groups ${ }^{\mathrm{a}}$

\begin{tabular}{lccc}
\hline & Age (y) & $\begin{array}{c}\text { ADC value }\left(\mathbf{m m}^{\mathbf{2}} / \mathbf{s e c} \times\right. \\
\left.\mathbf{1 0}^{-3}\right)\end{array}$ & Pvalue \\
\hline $\begin{array}{l}\text { Premenopausal } \\
\text { Group (n=106) }\end{array}$ & $34.82 \pm 7.7$ & $1.33 \pm 0.23$ & $<0.001$ \\
$\begin{array}{l}\text { Postmenopausal } \\
\text { Group (n= 55) }\end{array}$ & $54.10 \pm 7.4$ & $1.16 \pm 0.25$ & $<0.001$ \\
\hline
\end{tabular}

Abbreviations: ADC, apparent diffusion coefficient; SD, standard deviation. ${ }^{\mathrm{a}}$ Values are expressed as mean $\pm \mathrm{SD}$. 


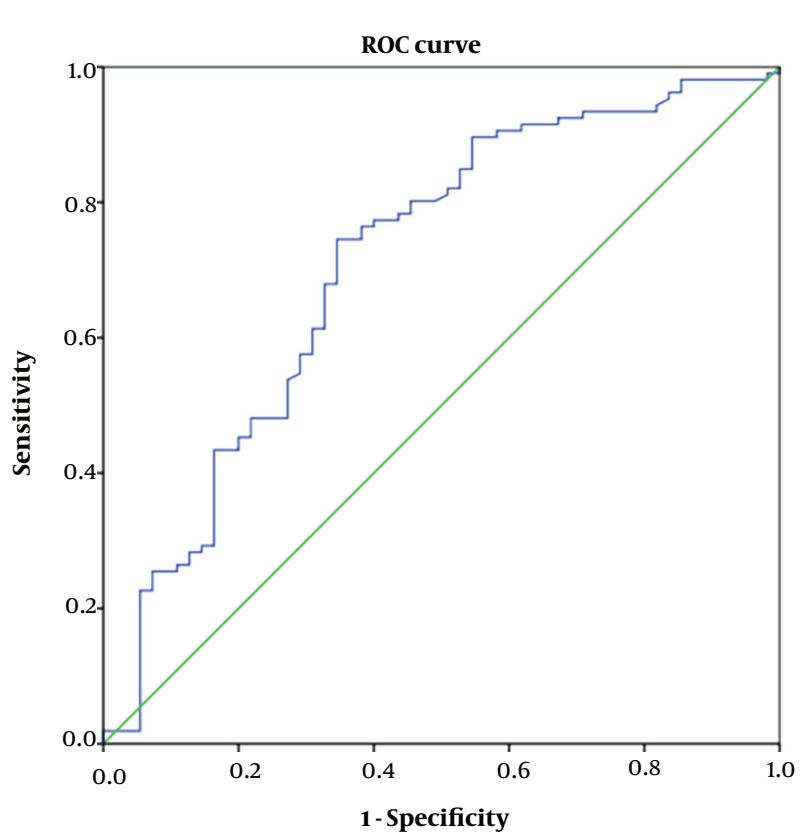

Figure 3. Receiver operator characteristics (ROC) curve of cervix apparent diffusion coefficient $(\mathrm{ADC})$ values.

\section{Discussion}

ADC value alterations with aging and menopausal status have been presented in this study. A negative correlation between aging and mean ADC values was revealed. Several studies showed age-related ADC value alterations in normal tissues such as the prostate, vertebra, and muscles (19-22). To our knowledge, no study has reported on age-related changes of cervix ADCs in the literature.

Multiple MRI parameters are associated with aging in muscles, and MRI can also be used to evaluate premature or abnormal aging of muscles (19). Yoon et al. showed that DWI could be used as an imaging tool to evaluate aging of tissues. In a study performed on children in 2018, it was shown that ADC values of the vertebra and intervertebral disks changed with the age groups and locations (20). Other studies revealed that ADCs of normal prostatic tissue changed by age, which could be a prognostic marker for evaluating prostate cancer $(21,22)$. In our study, we found that as age advanced, the cervix ADC value decreased (Figure 2), which may be pertinent to the evaluation of cervix lesions in premenopausal and postmenopausal women. In addition, the ICC of intraobserver consistency of ADC measurements was found as good in our study, which shows that it can be consistently used in measurements.

DWI, as a very popular and up-to-date imaging modality, that is being used widely, especially in gynecologic can- cers such as cervix and ovary cancers. It is also employed in the diagnosis of lung, prostate, kidney, and head and neck malignancies (23). In 2014, Zhao emphasized that DWI could be used for the differentiation of malignant epithelial and borderline ovarian tumors (24). In endometrial neoplasia, it has been reported that DWI can be used for the differentiation of malignant and benign endometrial tissues, as well as differentiation of endometrial serous and adenocancer (25-27).

Cervical cancer is one of the malignancies in which screening is performed effectively around the world. Today, precancerous lesions of the cervix are effectively treated with screening, and cervix screening provides early diagnosis. Imaging methods have an important role in the management of disease as well as in gynecologic examinations. Advanced imaging techniques are not recommended in the International Federation of Gynecologists and Obstetricians (FIGO) staging; however, imaging should be used appropriately to guide treatment (28). Several studies showed that ADC value, which is 1.50 $\mathrm{mm}^{2} / \mathrm{sec} \times 10^{-3}$ in the normal uterine cervix, was decreased in cervix cancer $(10,14)$. In study, the mean ADC value was $1.26 \mathrm{~mm}^{2} / \mathrm{sec} \times 10^{-3}$ in normal uterine cervix with $3 \mathrm{~T}$ MRI. In the study by Liu et al., the potential ability to differentiate between normal and cancerous tissue in the uterine cervix was shown (10). Therefore, the ADC value might be helpful in distinguishing benign, premalignant, and malignant tissue, as well as in gynecologic examinations and pathologic diagnosis. In our study, we did not include malignant and premalignant lesions; however, studies are needed to evaluate the effects of aging in cervix on malignant and premalignant lesions. Prospective studies that also include precancerous lesions of the cervix in a larger population are needed. For this reason, cervical ADC changes with age could be useful for clinical correlation.

DWI might be used in the diagnosis of cervical cancer in some suspicious cases, as well as in the management of cervical cancer. The current recommended treatment for early-stage cervical cancer is radical or modified radical hysterectomy (29). In addition to surgery, radiotherapy plays a critical role in the management of cervix carcinoma (30). DWI has been extensively used in the evaluation of the response to radiotherapy in cervix neoplasias. In a study in 2009 from China, it was shown that before treatment, ADCs for complete response were significantly lower than in partial response (12), suggesting that measurement of $\mathrm{ADC}$ values is important in cervical neoplasias. ADC values of tumors may also vary due to the age of the patient, because the ADC changes in the normal cervix with age. Studies are needed to evaluate if there are changes of ADC values of malignant lesions with age in the cervix. 
One of the main limitations of this study is that due to the retrospective design, the two groups consisted of an unequal number of subjects. Another limitation is that it did not include precancerous lesions.

In conclusion, the ADC values of the uterine cervix were inversely correlated with age, which is probably linked to alterations in cellular and extracellular matrix changes. This is the first study to show ADC value alterations with age and menopausal status.

\section{Footnotes}

Authors' Contribution: Study concept and design, acquisition of data, administrative, technical, material support, and study supervision: Funda Dinç Elibol; analysis and interpretation of data and statistical analysis: Sezen Bozkurt Köseoglu; drafting of the manuscript and critical revision of the manuscript for important intellectual content: Funda Dinç Elibol and Sezen Bozkurt Köseoglu

Conflict of Interests: None declared.

Ethical Approval: Ethical approval was obtained from the local Ethical Committee (code: 04/IV (31.05.2018)).

Financial Disclosure: None declared.

Funding/Support: No funding/support was received for this study.

Patient Consent: Informed consent to involve the study was waived due to the retrospective nature of the study.

\section{References}

1. Bammer R. Basic principles of diffusion-weighted imaging. Eur J Radiol. 2003;45(3):169-84. doi: 10.1016/s0720-048x(02)00303-0. [PubMed: 12595101].

2. Mascalchi M, Filippi M, Floris R, Fonda C, Gasparotti R, Villari N. Diffusion-weighted MR of the brain: Methodology and clinical application. Radiol Med. 2005;109(3):155-97. [PubMed: 15775887].

3. Schob S, Meyer J, Gawlitza M, Frydrychowicz C, Muller W, Preuss $M$, et al. Diffusion-weighted MRI reflects proliferative activity in primary CNS lymphoma. PLoS One. 2016;11(8). e0161386. doi 10.1371/journal.pone.0161386. [PubMed: 27571268]. [PubMed Central: PMC5003362].

4. Schob S, Surov A, Wienke A, Meyer HJ, Spielmann RP, Fiedler E. Correlation between aquaporin 4 expression and different DWI parameters in grade I meningioma. Mol Imaging Biol. 2017;19(1):138-42. doi 10.1007/s11307-016-0978-1. [PubMed: 27357591].

5. Chen L, Liu M, Bao J, Xia Y, Zhang J, Zhang L, et al. The correlation between apparent diffusion coefficient and tumor cellularity in patients: A meta-analysis. PLoS One. 2013;8(11). e79008. doi 10.1371/journal.pone.0079008. [PubMed: 24244402]. [PubMed Central: PMC3823989].

6. Noori M, Siahlou B, Salighehrad H, Fathi A, Fathi M. The importance of diffusion weighted imaging in breast MRI. Iran J Radiol. 2017;14(Special Issue). doi: 10.5812/iranjradiol.47964
7. Shi HF, Feng Q, Qiang JW, Li RK, Wang L, Yu JP. Utility of diffusionweighted imaging in differentiating malignant from benign thyroid nodules with magnetic resonance imaging and pathologic correlation. J Comput Assist Tomogr. 2013;37(4):505-10. doi: 10.1097/RCT.ob013e31828d28fo. [PubMed: 23863524].

8. Schob S, Voigt P, Bure L, Meyer HJ, Wickenhauser C, Behrmann $\mathrm{C}$, et al. Diffusion-weighted imaging using a readout-segmented, multishot EPI sequence at $3 \mathrm{~T}$ distinguishes between morphologically differentiated and undifferentiated subtypes of thyroid carcinoma-a preliminary study. Transl Oncol. 2016;9(5):403-10. doi: 10.1016/j.tranon.2016.09.001. [PubMed: 27661405]. [PubMed Central: PMC5035355].

9. Bonekamp S, Corona-Villalobos CP, Kamel IR. Oncologic applications of diffusion-weighted MRI in the body. J Magn Reson Imaging. 2012;35(2):257-79. doi:10.1002/jmri.22786. [PubMed: 22271274].

10. Liu Y, Bai R, Sun H, Liu H, Wang D. Diffusion-weighted magnetic resonance imaging of uterine cervical cancer. J Comput Assist Tomogr. 2009;33(6):858-62. doi: 10.1097/RCT.0b013e31819e93af. [PubMed: 19940650]

11. Kuang F, Ren J, Zhong Q, Liyuan F, Huan Y, Chen Z. The value of apparent diffusion coefficient in the assessment of cervical cancer. EurRadiol.2013;23(4):1050-8. doi:10.1007/s00330-012-2681-1.[PubMed: 23179520].

12. Liu Y, Bai R, Sun H, Liu H, Zhao X, Li Y. Diffusion-weighted imaging in predicting and monitoring the response of uterine cervical cancer to combined chemoradiation. Clin Radiol. 2009;64(11):1067-74. doi: 10.1016/j.crad.2009.07.010. [PubMed:19822239].

13. Liu Y, Liu H, Bai X, Ye Z, Sun H, Bai R, et al. Differentiation of metastatic from non-metastatic lymph nodes in patients with uterine cervical cancer using diffusion-weighted imaging. Gynecol Oncol. 2011;122(1):19-24. doi:10.1016/j.ygyno.2011.03.023. [PubMed: 21497384].

14. Liu Y, Ye Z, Sun H, Bai R. Clinical application of diffusion-weighted magnetic resonance imaging in uterine cervical cancer. Int J Gynecol Cancer. 2015;25(6):1073-8. doi: 10.1097/IGC.0000000000000472. [PubMed: 25966933].

15. Liu Y, Zhang Y, Cheng R, Liu S, Qu F, Yin X, et al. Radiomics analysis of apparent diffusion coefficient in cervical cancer: A preliminary study on histological grade evaluation. J Magn Reson Imaging. 2019;49(1):280-90. doi: 10.1002/jmri.26192. [PubMed: 29761595].

16. Winfield JM, Orton MR, Collins DJ, Ind TE, Attygalle A, Hazell $S$, et al. Separation of type and grade in cervical tumours using non-mono-exponential models of diffusion-weighted MRI. Eur Radiol. 2017;27(2):627-36. doi: 10.1007/s00330-016-4417-0. [PubMed: 27221560]. [PubMed Central: PMC5209433].

17. Exner M, Kuhn A, Stumpp P, Hockel M, Horn LC, Kahn T, et al. Value of diffusion-weighted MRI in diagnosis of uterine cervical cancer: A prospective study evaluating the benefits of DWI compared to conventional MR sequences in a $3 \mathrm{~T}$ environment. Acta Radiol. 2016;57(7):869-77. doi: 10.1177/0284185115602146. [PubMed: 26329683].

18. Kataoka M, Kido A, Koyama T, Isoda H, Umeoka S, Tamai K, et al. MRI of the female pelvis at 3T compared to 1.5T: Evaluation on highresolution T2-weighted and HASTE images. J Magn Reson Imaging. 2007;25(3):527-34. doi: 10.1002/jmri.20842. [PubMed: 17326081].

19. Yoon MA, Hong SJ, Ku MC, Kang CH, Ahn KS, Kim BH. Multiparametric MR imaging of age-related changes in healthy thigh muscles. Radiology. 2018;287(1):235-46. doi: 10.1148/radiol.2017171316. [PubMed: 29239712].

20. Tschischka A, Schleich C, Boos J, Eichner M, Schaper J, Aissa J, et al. Age-related apparent diffusion coefficients of lumbar vertebrae in healthy children at 1.5 T. Pediatr Radiol. 2018;48(7):1008-12. doi: 10.1007/s00247-018-4119-7. [PubMed: 29651606].

21. Tamada T, Sone T, Toshimitsu S, Imai S, Jo Y, Yoshida K, et al. Agerelated and zonal anatomical changes of apparent diffusion coefficient values in normal human prostatic tissues. J Magn Reson Imaging. 2008;27(3):552-6. doi: 10.1002/jmri.21117. [PubMed: 18219616]. 
22. Ren J, Huan Y, Wang H, Zhao H, Ge Y, Chang Y, et al. Diffusion-weighted imaging in normal prostate and differential diagnosis of prostate diseases.Abdom Imaging. 2008;33(6):724-8. doi:10.1007/s00261-008-93612. [PubMed: 18219519].

23. Surov A, Meyer HJ, Wienke A. Correlation between apparent diffusion coefficient (ADC) and cellularity is different in several tumors: A meta-analysis. Oncotarget. 2017;8(35):59492-9. doi:10.18632/oncotarget.17752. [PubMed: 28938652]. [PubMed Central: PMC5601748].

24. Zhao SH, Qiang JW, Zhang GF, Ma FH, Cai SQ, Li HM, et al. Diffusionweighted MR imaging for differentiating borderline from malignant epithelial tumours of the ovary: Pathological correlation. Eur Radiol. 2014;24(9):2292-9. doi: 10.1007/s00330-014-3236-4. [PubMed: 24871335].

25. Fujii S, Matsusue E, Kigawa J, Sato S, Kanasaki Y, Nakanishi J, et al. Diagnostic accuracy of the apparent diffusion coefficient in differentiating benign from malignant uterine endometrial cavity lesions: Initial results. Eur Radiol. 2008;18(2):384-9. doi: 10.1007/s00330-007-0769-9. [PubMed: 17917730].

26. Tian S, Liu A, Zhu W, Li Y, Chen L, Chen A, et al. Difference in diffusion-weighted magnetic resonance imaging and diffusion tensor imaging parameters between endometrioid endometrial adenocarcinoma and uterine serous adenocarcinoma: A retrospective study. Int J Gynecol Cancer. 2017;27(8):1708-13. doi: 10.1097/IGC.0000000000001054. [PubMed: 28945213].

27. Bharwani N, Miquel ME, Sahdev A, Narayanan P, Malietzis G, Reznek $\mathrm{RH}$, et al. Diffusion-weighted imaging in the assessment of tumour grade in endometrial cancer. Br J Radiol. 2011;84(1007):9971004. doi: 10.1259/bjr/14980811. [PubMed: 21896664]. [PubMed Central: PMC3473695].

28. Small WJ, Bacon MA, Bajaj A, Chuang LT, Fisher BJ, Harkenrider MM, et al. Cervical cancer: A global health crisis. Cancer. 2017;123(13):2404-12. doi: 10.1002/cncr.30667. [PubMed: 28464289].

29. Buchanan T, Pierce JY, Graybill W, Kohler M, Creasman W. Why do we continue to overtreat stage Ia carcinoma of the cervix? Am J Obstet Gynecol. 2017;217(4):413-7. doi: 10.1016/j.ajog.2017.05.020. [PubMed: 28522321].

30. de Souza Lawrence L. Radiation oncology management of stage I-III cervix cancer. Surg Oncol Clin N Am. 2017;26(3):477-89. doi 10.1016/j.soc.2017.01.002. [PubMed: 28576184]. 